\title{
A Collaborative VR Murder Mystery using Photorealistic User Representations
}

\author{
Ana Revilla* \\ The Modern Cultural \\ Bart Kevelham ${ }^{\dagger}$ \\ Artanim \\ Olivier Barbeau \\ Artanim
}

Jack Jansen

CWI

\author{
Sergio Zamarvide \\ The Modern Cultural \\ Valérie Juillard \\ Artanim \\ Caecilia Charbonnier \\ Artanim
}

Nacho Reimat

CWI

\author{
Ignacio Lacosta \\ The Modern Cultural \\ Brian Rochat \\ Artanim \\ Fernando Perez \\ The Modern Cultural \\ Michelle Drocco \\ Artanim
}

Patrick de Lange

Sound

Shishir Subramanyam

CWI
Jie Li

CWI
Yanni Mei Kinga Ławicka
CWI
Artanim

Javier Lajara

The Modern Cultural

Natasha Devaud

Pablo Cesar ${ }^{\S}$

CWI and TU Delft

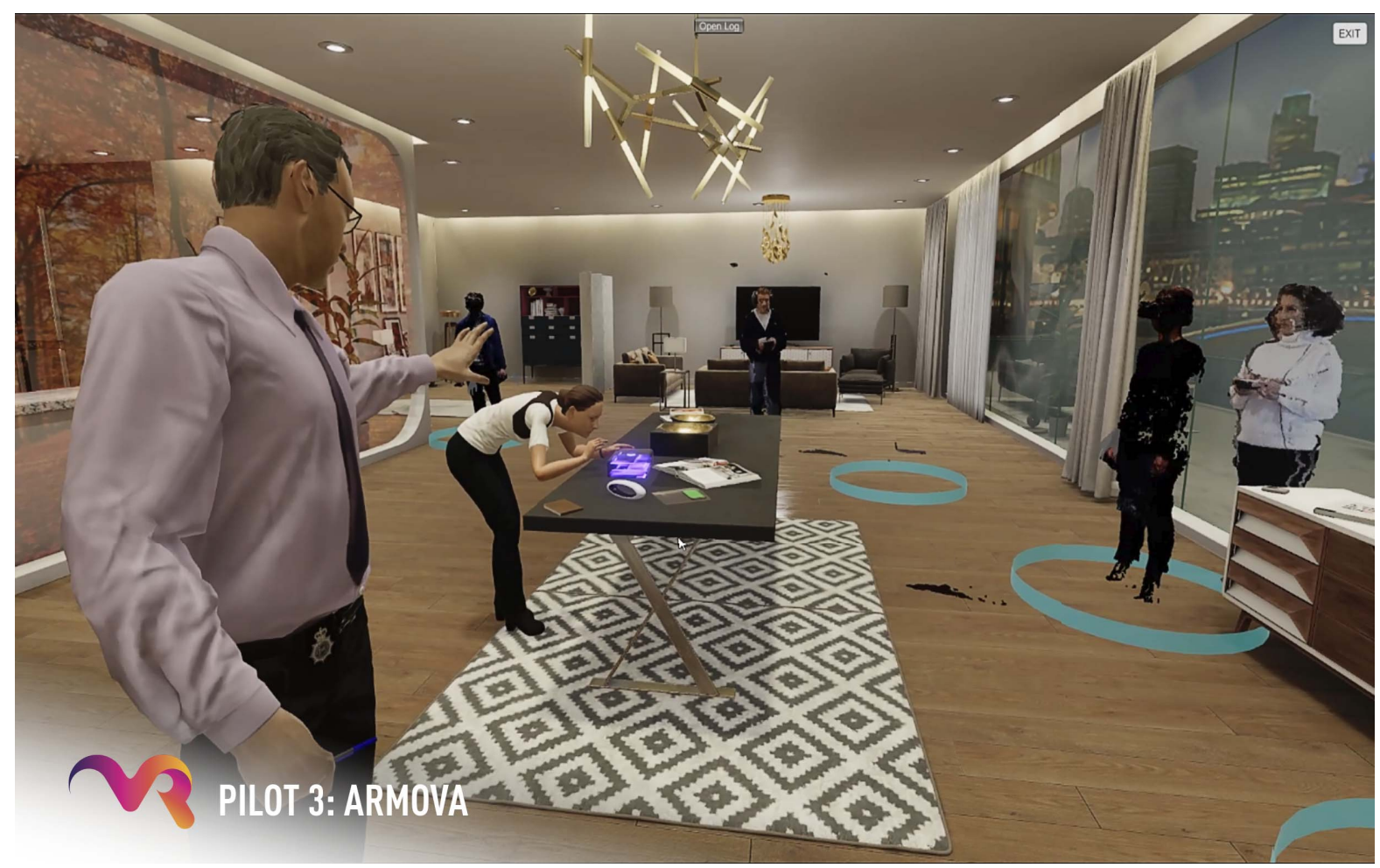

\section{Abstract}

The VRTogether project has developed a Social VR platform for remote communication and collaboration. The hyper-realistic representation of users, as volumetric video, allows for natural interaction in a virtual environment with others. This video shows one of the use cases, an escape room style, where remote users need to collaboratively resolve a murder mystery. The experience takes place in the victim's apartment where the police team (avatars) together with up to four real-time captured users (point clouds), work as a team to find clues and come up with a conclusion about what happened to the

\footnotetext{
*e-mail: anarevilla@themoderncultural.com

†e-mail: bart.kevelham@artanim.ch

*e-mail: patrick@sound.team

§e-mail: p.s.cesar@cwi.nl
}

victim and who was the criminal. This experience includes a layer of interaction, enabling the users to interact with the environment, by touching objects, and to talk to the characters. It also allows for navigating between the rooms of the apartment. The experience provides immersion and social connectedness, where users are protagonists of the story, sharing the virtual environment and following the narrative. The combination of virtual reality environments (space and characters) with novel technologies for real-time volumetric video conferencing enables unique new experiences in a number of areas such as healthcare, broadcasting, and gaming.

The video can be watched here: https://youtu.be/Hsj1YWo55k4

\section{ACKNOWLEDGMENTS}

This research work is funded by the European Commission as part of the H2020 program, under the grant agreement 762111, "VRTogether" (http://vrtogether. eu/). 